ASME Paper Number: 044501

Title: Using algebraic inequalities to reduce uncertainy and risk

DOI: $10.1115 / 1.4048403$

URL of paper after it is published on the ASME Digital Collection:

https://asmedigitalcollection.asme.org/risk/article-abstract/6/4/044501/1086898/Using-Algebraic$\underline{\text { Inequalities-to-Reduce-Uncertainty }}$ 


\title{
USING ALGEBRAIC INEQUALITIES TO REDUCE UNCERTAINTY AND RISK
}

\author{
Michael Todinov \\ School of Engineering, Computing and Mathematics \\ Oxford Brookes University, Oxford, UK \\ mtodinov@brookes.ac.uk
}

\begin{abstract}
The paper discusses applications of the domain-independent method of algebraic inequalities, for reducing uncertainty and risk. Algebraic inequalities are used for revealing the intrinsic reliability of competing systems and ranking the systems in terms of reliability in the absence of knowledge related to the reliabilities of their components. An algebraic inequality has also been used to establish the principle of the well-ordered parallel-series systems which, in turn, has been applied to maximise the reliability of common parallelseries systems.

The paper introduces a method of linking an abstract inequality to a real process by a meaningful interpretation of the variables entering the inequality and its left- and right-hand part. The meaningful interpretation of a simple algebraic inequality led to a counter-intuitive result. If two items from varieties are present in a large batch, the probability of selecting randomly two items of different variety does not exceed 0.5 .
\end{abstract}

Keywords: algebraic inequality, uncertainty reduction, risk reduction, domain-independent method

\section{Introduction}

Reliability and risk assessments in the case of uncertainty related to the values of riskcritical parameters still presents a great challenge to reliability engineering, risk management and decision making. In many cases, the actual values of the risk-critical parameters (e.g. material properties, dimensions, loads, magnitudes of the consequences of failure) are unknown or are associated with large uncertainty. However, almost all existing reliability analysis tools require reliability data which are unavailable at the design stage which makes the comparison of different design solutions very difficult. 
A formidable advantage of algebraic inequalities is that they do not require knowledge related to the distributions of the variables entering the inequalities. This makes the method based on algebraic inequalities ideal for handling deep uncertainty associated with components, properties and control parameters and for ranking designs in the absence of reliability data related to the separate components. The method of algebraic inequalities does not rely on reliability data or detailed knowledge of physical mechanisms underlying possible failure modes. This is why, the method is appropriate for new designs, with no failure history and with insufficiently researched failure mechanisms.

By proving algebraic inequalities related to the reliabilities of competing systems/processes, it is possible to reveal their intrinsic reliability and rank them in terms of reliability in the absence of knowledge related to the reliabilities of their building parts.

Another formidable advantage of the algebraic inequalities is their capacity to reduce aleatory and epistemic uncertainty and produce tight upper and lower bounds related to uncertain reliability-critical design parameters such as material properties, dimensions, loads and component reliabilities.

Yet another formidable advantage of algebraic inequalities is that they admit a meaningful interpretation which can be attached to a real system or process. To the best of our knowledge, creating a meaningful interpretation for existing non-trivial abstract inequalities and attaching it to a real system or process has not yet been explored and this is also in focus of this paper.

Algebraic inequalities, have been used extensively in mathematics and a number of useful non-trivial algebraic inequalities have been well documented [1-8]. A useful overview of the use of inequalities in mathematics has been presented in [7].

In reliability and risk research, inequalities have been used exclusively as a tool for reliability and risk evaluation and for characterisation of reliability functions [9-16]. However, the reliability-related applications of inequalities are very limited and oriented towards measuring the reliability performance of the systems, instead of providing direct input to the design process by reducing uncertainty and risk and improving the reliability of components, systems and processes.

Although standard reliability textbooks [17-20] do allocate space on reliability improvement methods such as introducing redundancy, derating, eliminating common cause and condition monitoring, there is a clear lack of discussion related to improving reliability by using algebraic inequalities. 
Applications of inequalities have also been considered in physics [21] and engineering [22-23]. However, in the mechanical engineering design literature [23-31], there is also a lack of discussion on the use of algebraic inequalities to improve reliability and reduce risk. In engineering design, the application of inequalities is mainly confined to trivial inequalities linking design variables required to satisfy various trivial design constraints in order to guarantee that the design will perform its required functions [23].

To the best of our knowledge of existing approaches involving inequalities in reliability and risk research, algebraic inequalities have not yet been used as a domain-independent reliability improvement or risk reduction method which makes use of the capability of the inequalities to rank systems and processes in the absence of knowledge related to the values of the controlling variables.

It is only recently that work related to applying advanced algebraic inequalities for uncertainty and risk reduction was published [32]. The present paper extends this work by using inequalities for ranking systems with unknown reliabilities of their components. In addition, the paper introduces a method of linking an abstract inequality to a real process by a meaningful interpretation of the variables entering the inequality and its parts.

\section{Using inequalities for ranking systems with components with unknown reliabilities}

Comparing the performance of alternative systems has always been an essential element of the economic analysis and the basis for making an informed choice [33].

Often, the reliabilities of the components building the systems are unknown. As a result, the epistemic uncertainty associated with the reliabilities of the components building the system translates into epistemic uncertainty related to which system has superior reliability.

An important way of using inequalities to improve reliability and reduce risk is to start with the competing real systems/processes and derive and prove an algebraic inequality which ranks their performance.

For two competing systems (a) and (b) built on components whose reliabilities are unknown, the steps that lead to the elimination of the uncertainty about which system is superior can be summarized as follows.

- For each of the competing systems build the reliability network from its functional diagram.

- By using methods from system reliability analysis, determine the system reliabilities $R_{a}$ and $R_{b}$ of the systems or the probabilities of system failure $F_{a}$ and $F_{b}$. 
- Subtract the reliabilities of the competing systems or the probabilities of system failure and test and prove any of the inequalities: $R_{a}-R_{b}>0, R_{a}-R_{b}<0 \quad F_{a}-F_{b}>0, F_{a}-F_{b}<0$.

Very often, in comparing the performance of two competing systems, knowledge about the reliability of the components is missing. Only knowledge about the age ranking of the components is available. Such is the case where new components, old-age and medium-age components of the same type are used in the same system. Because most of the components fail due to damage accumulation (wearout), it is correct to assume that the new components are more reliable than the medium-age components and the medium-age components are more reliable than the old-age components.

Consider the functional diagrams of three systems built with pipes and four valves $(A, B, C$ and $D$ ) with different ages (Figure 1). The valves are working independently from one another and all of them are initially open. The question of interest is which is the most reliable system with respect to the function 'stopping the flow of fluid in the pipeline'. The signal for closing is issued to all valves simultaneously.

Valve $A$ is a new valve, followed by valves $B$ and $C$ with intermediate age. Valve $D$ is an old valve. The reliabilities of the valves are denoted by $a, b, c$ and $d$. With respect to the function 'valve closure on demand', depending on their age, the reliabilities of the valves can be ranked as follows: $a>b>c>d$.

Figure 1. Three possible arrangements involving four different types of valves

It has been conjectured that the arrangement in Figure 1c is superior to the arrangements in Figures $1 \mathrm{a}$ and $1 \mathrm{~b}$.

The reliability networks (reliability block diagrams) of the systems with respect to the function 'stopping the fluid in the pipeline on command' are given in Figure 2. The reliability networks show the logical arrangement of the valves with respect to the function 'stopping the fluid in the pipeline on command'.

The reliabilities of systems (a), (b) and (c) are given by

$$
\begin{gathered}
R_{a}(a, b, c, d)=[1-(1-a)(1-b)] \times[1-(1-c)(1-d)]=(a+b-a b)(c+d-c d) \\
R_{b}(a, b, c, d)=[1-(1-a)(1-c)] \times[1-(1-b)(1-d)]=(a+c-a c)(b+d-b d) \\
R_{c}(a, b, c, d)=[1-(1-a)(1-d)] \times[1-(1-b)(1-c)]=(a+d-a d)(b+c-b c)
\end{gathered}
$$

Proving that configuration (c) is superior reduces to proving the inequalities 


$$
R_{c}(a, b, c, d)>R_{a}(a, b, c, d) \text { and } R_{c}(a, b, c, d)>R_{b}(a, b, c, d)
$$

which are equivalent to the inequalities

$$
\begin{aligned}
& (a+d-a d)(b+c-b c)-(a+b-a b)(c+d-c d)>0 \\
& (a+d-a d)(b+c-b c)-(a+c-a c)(b+d-b d)>0
\end{aligned}
$$

Figure 2. Reliability networks of the systems in Figure 1 with respect to the function 'stopping the fluid in the pipeline on command.

Manipulating the left-hand side of inequality (4) results in

$$
\begin{aligned}
& (a+d-a d)(b+c-b c)-(a+b-a b)(c+d-c d) \\
& \quad=a b+d c-a d-b c=a(b-d)-c(b-d)=(a-c)(b-d)
\end{aligned}
$$

Considering that $a>b>c>d$, it follows that $(a-c)(b-d)>0$. Therefore, configuration (c) is more reliable than configuration (a). Manipulating the left hand side of inequality (5) results in

$$
\begin{aligned}
& (a+d-a d)(b+c-b c)-(a+c-a c)(b+d-b d) \\
& \quad=a c+d b-a d-c b=a(c-d)-b(c-d)=(a-b)(c-d)
\end{aligned}
$$

Considering that $a>b>c>d$, it follows that $(a-b)(c-d)>0$. Therefore, configuration (c) is more reliable than configuration (b). Consequently, configuration (c) is the most reliable configuration.

Consider now the functional diagrams of four possible configurations built with pipes and valves of the same type: new valves $A$ and old valves $B$ (Figure 3 ). With respect to the function 'valve closure on command', a new valve $A$ is more reliable than an old valve $B$. If the reliabilities of the valves are denoted by $a$ and $b$, the inequality $a>b$ holds.

The valves are working independently from one another and all of them are initially open. The question of interest is which is the most reliable configuration with respect to the function 'stopping the fluid in the pipeline on command'. The signal for closure is issued to all valves simultaneously.

The reliability networks of the systems with respect to the function 'stopping the fluid in the pipeline', are given in Figure 4.

Figure 3. The same system topology built with new valves $A$ and old valves $B$. 
Figure 4. Reliability networks of the systems in Figure 3 with respect to the function 'stopping the fluid in the pipeline on command'.

The reliabilities of systems (a)-(d) are given by

$$
\begin{aligned}
& R_{a}(a, b)=\left[1-\left(1-a^{2}\right)(1-a b)\right] b^{2}=a b^{2}\left(a+b-a^{2} b\right) \\
& R_{b}(a, b)=\left[1-(1-a b)\left(1-b^{2}\right] a^{2}=a^{2} b\left(a+b-a b^{2}\right)\right. \\
& R_{c}(a, b)=[1-(1-a b)(1-a b)] a b=a^{2} b^{2}(2-a b) \\
& R_{d}(a, b)=\left[1-\left(1-a^{2}\right)\left(1-b^{2}\right)\right] a b=a b\left(a^{2}+b^{2}-a^{2} b^{2}\right)
\end{aligned}
$$

It is conjectured that system ' $b$ ' is the most reliable system. For the differences of the system reliability characterising configuration $(b)$ and the system reliability characterising the rest of the configurations, the following relationships hold:

$$
\begin{aligned}
& R_{b}(a, b)-R_{a}(a, b)=a b\left(a^{2}-b^{2}\right)>0 \\
& R_{b}(a, b)-R_{c}(a, b)=a^{2} b(a-b)>0 \\
& R_{b}(a, b)-R_{d}(a, b)=a b^{2}(a-b)>0
\end{aligned}
$$

The configuration (b) is indeed characterised by the highest reliability.

In the examples considered in this section, the algebraic inequalities helped to reveal the intrinsic reliability of the competing design solutions and rank the systems in terms of reliability in the absence of knowledge related to the reliabilities of their building parts.

\section{Maximising the reliability of parallel-series systems by using an algebraic inequality}

Consider the system in Figure 5 which transports cooling fluid from three sources s1,s2 and s3 to a chemical reactor $C R$. The cooling system consists of identical pipeline sections (the rectangles in Figure 5) working independently from each other. Each pipeline section includes a pump for transporting the cooling fluid through the section. Suppose that all pipeline sections and the pumps are old (sections ' $O$ ' in Figure 5) and prone to damage accumulation due to fatigue, wear, deteriorated seals, etc. Cooling of the chemical reactor is done successfully if at least one cooling line (including three cooling sections) delivers cooling fluid to the reactor.

Suppose that there is opportunity (budget) to replace three old cooling sections with new ones. This can be done different ways, one of which is shown in Figure $5 \mathrm{~b}$ (the new sections 
have been marked by ' $n$ ' in Figure 5). The question of interest is replacement of which sections maximises the reliability of the system (the probability of uninterrupted cooling of the reactor). Without detailed analysis it seems that the replacement of any three sections would have the same effect. However, this conclusion is incorrect. Only the replacement of three sections from the same cooling line maximises the reliability of the system, compared to any other replacement.

The solution maximising the reliability of the system creates a well-ordered parallel-series system. The well-ordered parallel-series system is obtained if the available components are used first to build the branch with the highest possible reliability; next, the remaining components are used to build the branch with the second-highest reliability and so on.

Here, it needs to be pointed out that the systems of the type given in Figure 5 are very common. Consider detectors D1, D2 and D3 (Figure 6) coupled with sensors for detecting the increase of a particular safety-critical parameter (temperature, pressure, humidity, concentration displacement, etc.). The components in each detector are usually logically arranged in series (Figure 6). This means that the detector will only work (will detect the increase of the parameter) if all building elements (blocks) of the detector work. In order to detect the increase of the safety-critical parameter, at least one of the detectors must detect the increase of the parameter. Consequently, with respect to detecting the increase of the critical parameter, the detectors are logically arranged in parallel.

The well-ordered parallel-series system is characterised by the smallest possible risk of failure.

Proof. This property will be proved by making use of an inequality. Suppose that there is a system that is not well-ordered and which possesses the highest possible reliability. The branches in a parallel-series system can always be re-arranged in such a way that for any two branches ' $i$ ', ' $j$ ' for which $i<j$, the branch with index ' $i$ ' is equally reliable or more reliable than branch ' $j$ ' ( $\left.R_{i} \geq R_{j}\right)$, where $R_{i}$ and $R_{j}$ are the reliabilities of branch $i$ and branch $j$, respectively. If the system is not a well-ordered system, then there will be two branches $a$ and $b(a<b)$ with reliabilities $R_{a} \geq R_{b}$, where there will be at least one component in branch $b$ with a larger reliability than the reliability of the analogous interchangeable component in branch $a$. Suppose that $R_{a}=a_{1} a_{2} \times \ldots \times a_{n a}$ and $R_{b}=b_{1} b_{2} \times \ldots \times b_{n b}$ are the reliabilities of branches $a$ and $b$ and $n a, n b$ are the number of components in branches $a$ and $b$, correspondingly. Without loss of generality, suppose that the two interchangeable 
components are the last components in the branches $a$ and $b\left(a_{n a}<b_{n b}\right)$. The reliability of the initial system can be presented as

$$
R_{\text {sys } 1}=1-\left(1-a_{1} a_{2} \times \ldots \times a_{n a}\right) \times\left(1-b_{1} b_{2} \times \ldots \times b_{n b}\right) \times\left[1-R_{\text {rest }}\right]
$$

where $R_{\text {rest }}$ is the reliability of the rest of the parallel-series arrangement (excluding branches $a$ and $b$ ). After swapping components $a_{n a}$ and $b_{n b}$, the reliability of the resultant system becomes

$$
R_{\text {sys } 2}=1-\left(1-a_{1} a_{2} \times \ldots \times a_{n a-1} b_{n b}\right) \times\left(1-b_{1} b_{2} \times \ldots \times b_{n b-1} a_{n a}\right) \times\left[1-R_{\text {rest }}\right]
$$

Subtracting (14) from (13) yields:

$$
R_{s y s 1}-R_{\text {sys } 2}=\left(a_{n a}-b_{n b}\right) \times\left[1-R_{\text {rest }}\right] \times\left(a_{1} a_{2} \times \ldots \times a_{n a-1}-b_{1} b_{2} \times \ldots \times b_{n b-1}\right)
$$

Figure 5. A safety-critical cooling system consisting of three cooling lines, delivering cooling fluid to a chemical reactor.

Figure 6. A safety-critical system consisting of three detectors working in parallel.

The inequality

$$
R_{a}=a_{1} a_{2} \times \ldots \times a_{n a} \geq R_{b}=b_{1} b_{2} \times \ldots \times b_{n b}
$$

holds by the way the branches have been arranged in descending order, according to their reliability $\left(R_{a} \geq R_{b}\right)$ and because $a_{n a}<b_{n b}$ (by assumption). Dividing the left hand side of (16) by the positive value $a_{n a}$ and the right hand side of (16) by the larger positive value $b_{n b}$, yields the stronger inequality

$$
a_{1} a_{2} \times \ldots \times a_{n a-1}>b_{1} b_{2} \times \ldots \times b_{n b-1}
$$

which means that in equation (15):

$$
a_{1} a_{2} \times \ldots \times a_{n a-1}-b_{1} b_{2} \times \ldots \times b_{n b-1}>0
$$

holds. Since $1-R_{\text {rest }}>0$ and $a_{n a}-b_{n b}<0$, the right hand side of equation (15) is negative, which means that the resultant system (after the swap of two components of the same type) has a higher reliability. This contradicts the assumption that the initial system (which is not well ordered) possesses the highest possible reliability. It was thus demonstrated that the reliability of a system which is not well-ordered, can be improved by swapping components between parallel branches. A well-ordered system is unique and there can be no two well- 
ordered systems. Because a parallel-series system can either be a well-ordered or not a wellordered system, the well-ordered system has the highest reliability.

In cases, where a possibility for a permutation of components with similar functions exists, the permutation should be done in such a way that a branch with the highest possible reliability is built first by using the possible permutations, followed by using the remaining possible permutations to build a branch with the next highest possible reliability and so on. This can be illustrated with maximising the reliability of the cooling system in Figure 7 which includes sections with different levels of deterioration. The system includes new sections ('n'), medium-age sections ('m') and old sections ('o'). It is assumed that the new sections are associated with the highest reliability and the old sections are associated with the lowest reliability. The reliability $R(m)$ of the medium-age sections is ranked between the reliability $R(n)$ of the new sections and the reliability $R(o)$ of the old sections. This is expressed by the inequalities:

$$
R(n)>R(m)>R(o)
$$

Since the sections of the cooling systems are interchangeable, a permutation of the sections can be made such that a cooling branch with the highest possible reliability is built first by swapping the old and medium-age sections from the first branch with new sections. The result is shown in Figure 7b. Next, a cooling branch with the next highest reliability is built with the remaining sections by swapping the old sections from the second branch with medium-age sections from the third branch. The result is shown in Figure 7c, which is a wellordered system possessing the highest reliability.

Figure 7. Maximising the reliability of a parallel-series system including components with different levels of deterioration

Permutation of interchangeable components with different level of deterioration, offers the valuable opportunity to maximise the reliability of systems without any knowledge related to the reliabilities of the separate components. Unlike traditional approaches, most of which require resources to improve reliability, the method of permutation, based on the rearrangement inequality, is not associated with extra cost.

This method can also be used for determining the optimal locations of condition monitoring equipment which maximise system reliability. Consider the cooling system from Figure 8a, which includes old sections only. The presence of a condition monitoring equipment on an old cooling section increases the reliability of the section because it permits 
detecting deteriorated performance of a pump or a filter and replacing them before failure or blockage occur. Assume that only few pieces of condition monitoring equipment are available for monitoring the condition of the pumps/filters in the cooling sections.

Because of cost limitations, it is not possible to install condition monitoring on all cooling sections. Suppose that, due to budget constraints, the number of condition monitoring devices is limited to three. The question of interest is the optimal locations of the condition monitoring equipment which maximise the reliability of the cooling system.

Considering the previous discussion, to maximise the reliability of the system, the condition monitoring devices must all be located along a single cooling branch (Figure $8 \mathrm{~d}$ ). According to the principle of the well-ordered parallel-series systems, this is equivalent to building first a branch with the highest possible reliability.

The optimal locations of condition monitoring equipment provides the right balance between cost, risk and performance which translates into low downtimes, high network availability and small maintenance costs.

Figure 8. Optimal condition monitoring of the sections of the cooling system.

\section{Meaningful interpretation of an abstract algebraic inequality}

There is an alternative way of using algebraic inequalities for optimisation, uncertainty and risk reduction. This method consists of moving in the opposite direction: starting with an existing abstract inequality and moving towards the real system or process. An important step of this very powerful approach is creating relevant meaning for the variables entering the algebraic inequality, followed by a meaningful interpretation of the left- and right-hand side of the inequality which links it with a real physical system or process.

Consider the common abstract inequality

$$
a^{2}+b^{2} \geq 2 a b
$$

which is true for any real numbers $a$ and $b$ because the inequality can be obtained directly from the obvious inequality $(a-b)^{2} \geq 0$. Suppose that the numbers $a$ and $b$ are not both equal to zero. If both sides of inequality (18) are divided by the positive quantity $(a+b)^{2}$, the next inequality 


$$
\frac{a^{2}}{(a+b)^{2}}+\frac{b^{2}}{(a+b)^{2}} \geq \frac{2 a b}{(a+b)^{2}}
$$

is obtained which has a powerful interpretation. If $a$ and $b$ are the number of items of variety $A$ and variety $B$ in a large batch of items, the left side of inequality (19) is the probability that from two randomly selected items from the batch, both will be of the same variety (variety $A$ only or variety $B$ only).

The varieties can, for example, be components manufactured by two different machine centres. It is assumed that the batch is sufficiently large so that if two items are taken from the batch, the probability that the second item will be of a particular variety is practically independent of the variety of the first item.

The right side of inequality (19) can be interpreted as the probability that the two randomly selected items, will be of different variety $(A, B$ or $B, A)$. The two selected components can either be of the same variety or of a different variety. Therefore, the sum of the probability of the event: 'the components are of the same variety' and the probability of the event 'the components are of different variety' add up to unity. Consequently, considering inequality (19), the probability that the two selected items will be of different variety is smaller than 0.5 or at most equal to 0.5 but never exceeds 0.5 . This conclusion is counter-intuitive, considering the symmetry of the cases leading to the same-variety outcome and to a different-variety outcome. Thus, because of symmetry, flipping two identical coins is equally likely to result in the same outcome on both coins (head/head or tail/tail) or in different outcomes on the coins (head/tail or tail/head).

The difference between the probabilities calculated from the left-hand side and the righthand side of inequality (19) can be very large. Thus, for $a=300$ items of variety $A$ and 800 items the left hand side yields probability of 0.603 for selecting two components of the same variety and probability of 0.397 that the selected components will be of different variety.

Interestingly, no analogous inequality exists for a larger than two number of randomly selected components from a large batch containing two different varieties. Thus, the probability of selecting $n$ components of the same variety $(n>2)$ from a large batch ( $n<<a+b)$ is given by

$$
p_{1}=a^{n} /(a+b)^{n}+b^{n} /(a+b)^{n}
$$

while the probability of not selecting three components of the same variety is $p_{2}=1-p_{1}$ as complementary events. 
Denoting $u=a /(a+b)$ and substituting in (20) yields $p_{1}=u^{n}+(1-u)^{n}$. Let us conjecture that the inequality $p_{1} \geq p_{2}$ holds for $n>2$. This conjecture is equivalent to the conjectured inequality

$$
u^{n}+(1-u)^{n} \geq 1 / 2
$$

The maximum of the expression $x^{n}+y^{n}$ where $x+y=1$ is obtained when $x=y=1 / 2$. Consequently, the maximum of the left hand side of (21) is obtained when $u=1-u$ or when $u=1 / 2$. However, $(1 / 2)^{n}+(1 / 2)^{n}<1 / 2$ for any $n>2$. The only value for which the equality in (21) is attained is $n=2$. As a result, Inequality (21) does not hold for any $n>2$.

Now, let us conjecture that the inequality $p_{1} \leq p_{2}$ holds for any $n>2$. This is equivalent to the inequality

$$
u^{n}+(1-u)^{n} \leq 1 / 2
$$

The conjectured inequality (22) can be disproved by providing a counterexample. For example, the inequality is not correct for $n=3$ and $u=0.9$. By taking $u$ sufficiently close to unity, inequality (22) can be disproved for any other $n>2$.

Inequality (19) has important applications. Increasing the level of balancing in a system is an important domain-independent method for reliability improvement and risk reduction.

Selecting two items of the same variety is an important condition for reducing variability and guaranteeing that that the system (where the components are assembled) is well-balanced and with high-reliability. As a rule, well-balanced systems distribute the load more uniformly across components and exhibit higher reliability. The requirement for the same variety components ensuring a more uniform distribution of the load is essential for both electrical and mechanical components: for example, for transistors in power supply circuits, mechanical components in contact, resistors in sensitive bridge circuits, bearings in rotating shafts, belts in belt drives, etc. Thus, selecting transistors of the same variety working in parallel in a power supply circuit is an important measure to ensure a uniform distribution of the load on the transistors and avoid overheating and premature failure of any of the transistors.

For mechanical components in contact, selecting both components of the same variety (for example, both with high surface hardness or both with normal surface hardness) is an important measure to reduce the rate of wear and avoid premature wear out of one of the components. 


\section{Conclusions}

1. By using algebraic inequalities, competing systems have been be ranked successfully in terms of reliability in the absence of knowledge about the reliabilities of their components.

2. Meaningful interpretation of the variables and the left and right hand side of an abstract algebraic inequality can be used to link the inequality to a real process. The meaningful interpretation of a simple algebraic inequality led to a counter-intuitive result. If items from two varieties are present in a large batch, the probability of randomly selecting two items of different variety does not exceed 0.5. Interestingly, no analogous result exists for a larger than two number of randomly selected components.

3. Algebraic inequality has been used to establish the principle of the well-ordered parallelseries systems which was used to maximise the reliability of common parallel-series systems.

\section{REFERENCES}

1. Kazarinoff N.D. Analytic Inequalities. New York: Dover Publications, Inc., 1961.

2. Bechenbach E and Bellman R. An introduction to inequalities, New York: Random, 1961

3. Engel A., Problem-solving strategies. Springer: New York, 1998.

4. Hardy G, Littlewood JE and Pólya G. Inequalities. Cambridge Mathematical Library, Cambridge University Press, 1999.

5. Steele J.M. The Cauchy-Schwarz master class: An introduction to the art of mathematical inequalities. New York: Cambridge University Press, 2004.

6. Mitrinovic D.S., Analytic Inequalities, Springer-Verlag, New York/Berlin. 1970.

7. Fink A.M., An essay on the history of inequalities, Journal of Mathematical Analysis and Applications, 249, 118-134 (2000)

8. Pop O. About Bergström's inequality, Journal of Mathematical Inequalities, 3 (2), 237242, 2009.

9. Ebeling CE. Reliability and maintainability engineering. McGraw-Hill: Boston, 1997.

10. Xie M and Lai CD. On reliability bounds via conditional inequalities, Journal of Applied Probability, 35(1), 104-114, 1998. 
11. Makri FS and Psillakis ZM. Bounds for reliability of k-within two-dimensional consecutive-r-out-of-n failure systems, Microelectronics Reliability, 36(3), 341-345, 1996.

12. Hill S.D., Spall J.C. and Maranzano C.J. Inequality-Based Reliability Estimates for Complex Systems, Naval Research Logistics, 367-374, 2013, DOI 10.1002/nav.

13. Berg VD and Kesten H. Inequalities with Applications to Percolation and Reliability, Journal of Applied Probability, 22(3):556-569, 1985.

14. Kundu $\mathrm{C}$ and Ghosh $\mathrm{A}$. Inequalities involving expectations of selected functions in reliability theory to characterize distributions, Communications in Statistics - theory and methods, 46(17), 2017, https://doi.org/10.1080/03610926.2016.1183784.

15. Dohmen K. Improved Inclusion-exclusion identities and Bonferoni inequalities with reliability applications, SIAM J. Discrete Math., 16(1), 156-171, 2006.

16. DeGroot MH. Probability and statistics, 2nd ed. Reading: Addisson-Wesley Publishing Company, 1986.

17. Lewis, EE. Introduction to Reliability Engineering. New York:Wiley, 1966.

18. O’Connor, PDT. Practical Reliability Engineering, 4e. New York: Wiley, 2002.

19. Dhillon BS. Engineering systems reliability, safety, and maintenance. Boca Raton, New York: CRC Press, 2017.

20. Modarres M, Kaminskiy MP, Krivtsov V. Reliability engineering and risk analysis, a practical guide, 3rd ed. CRC Press, 2017.

21. Rastegin A. Convexity inequalities for estimating generalized conditional entropies from below. Kybernetika, 48(2): 242-253, 2012.

22. Cloud M, Byron C, Lebedev LP. Inequalities: with applications to engineering. SpringerVerlag: New York, 1998.

23. Samuel A and Weir J. Introduction to engineering design: Modelling, synthesis and problem solving strategies. Elsevier: London, 1999.

24. Collins JA. Mechanical design of machine elements and machines. John Wiley \& Sons, Inc.: New York, 2003.

25. Norton RL. Machine design, an integrated approach, 3rd ed. Pearson International edition., 2006.

26. Pahl G, Beitz W, Feldhusen J and Grote KH. Engineering design. Springer: Berlin, 2007.

27. Childs PRN. Mechanical design engineering handbook. Elsevier: Amsterdam, 2014.

28. Budynas RG, Nisbett JK. Shigley's Mechanical engineering design, 10th ed. McGrawHill Education, 2015. 
29. Mott RL, Vavrek EM, Wang J. Machine Elements in Mechanical Design, 6th ed. Pearson Education Inc., 2018.

30. Gullo LG, Dixon J. Design for safety. Wiley: Chichester, 2018.

31. French M. Conceptual design for engineers. 3rd ed. Springer-Verlag Ltd: London, 1999.

32. Todinov MT. Improving reliability and reducing risk by using inequalities, Safety and reliability, 2019, doi.org/10.1080/09617353.2019.1664129.

33. Todinov, M.T. Reliability analysis of complex systems based on the losses from failures, International Journal of Reliability, Quality and Safety, 13(2), 127-148, 2006. 